\title{
LE PALEOENVIRONNEMENT VEGETAL DES OCCUPATIONS HUMAINES DE LA GROTTE DE PAYRE A LA FIN DU PLEISTOCĖNE MOYEN ET AU DÉBUT DU PLEISTOCÈNE SUPÉRIEUR (ARDĖCHE, FRANCE)
}

\author{
VEGETAL PALEOENVIRONMENT IN THE HUMAN SETTLEMENTS FOUND IN PAYRE \\ CAVE AT THE END OF THE MIDDLE PLEISTOCENE AND THE BEGINNING OF THE \\ UPPER PLEISTOCENE (ARDECHE, FRANCE)
}

CHAFIKA KALAI $(*)$

MARIE-HELÈNE MONCEL $(*)$

JOSETTE RENAULT-MIKOVSKY $(*)$

\begin{abstract}
RESUMÉ
Le gisement paléolithique moyen de Payre est situé dans le sud-est de la France, dans la moyenne vallée du Rhône en contexte méditerranéen. Les fouilles, qui s'y déroulent depuis 1990, livrent une séquence datée des stades isotopiques 7 à 5. L'étude palynologique des niveaux d'occupation des stades isotopiques 6 et 5 nous informe sur le contexte végétal de la fin du Pléistocène moyen et du début du Pléistocène supérieur. Le paysage est, lors des diverses occupations humaines, globalement semi-ouvert et le climat de type tempéré sous influence méditerranéenne.
\end{abstract}

\begin{abstract}
The Middle Palaeolithic site named Payre is located in the south-east of France, in the Middle Rhone Valley, in the mediterranean world. Since 1990, the excavations have yielded a sequence dated from the isotopic stages 7 to 5. The palynological study based on settlement levels from the isotopic stages 6 and 5 has provided us with information about the vegetal environment of the end of the Middle Pleistocene and the beginning of the Upper Pleistocene. When men came, the landscape was semi-forest and the climate was temperate with mediterranean influences.
\end{abstract}

(*) Laboratoire de Préhistoire. CNRS. UMR 6569. Muséum National d'Histoire Naturelle. Institut de Paléontologie Humaine, 1 rue René Panhard, 75013 Paris, France. Tél. : 0143316291. Fax: 01433122 79. Email: moncel@mnhn.fr

El artículo fue remitido en su versión el 24-X-2000.

\section{RESUMEN}

El emplazamiento arqueológico del Paleolítico Medio de Payre se sitúa al sureste de Francia, en el valle del Ródano, en el contexto mediterráneo actual. Las excavaciones que vienen llevándose a cabo desde 1990 presentan una secuencia fechada en los estadios isotópicos 7 al 5. El estudio palinológico de los niveles de ocupación de los estadios isotópicos 6 y 5 nos informan sobre el contexto vegetal del final del Pleistoceno Medio y de principios del Pleistoceno Superior. Los diferentes periodos climáticos se caracterizan por la predominancia de los taxones arbóreos como Quercus t. ilex y Buxus. El paisaje es a lo largo del diagrama, globalmente semi-abierto y el clima de tipo templado presenta influencias mediterráneas.

Mots-clé: Paleoenvironnement de l'homme fossile. Palynologie. Paléolithique moyen. Stades isotopiques 7 à 5 . Ardèche. France.

Key words: Palaeoenvironment. Palynology. Middle Palaeolithic. Isotopic stages 7 to 5. Ardèche. France.

Palabras clave: Palinología. Paleolítico Medio. Estadios isotópicos 7 al 5. Ardèche. Francia.

\section{INTRODUCTION}

Le site paléolithique moyen de Payre est l'un des rares gisements de la moyenne vallée du Rhône datés des stades isotopiques 7 à 5 . Mis à part les 


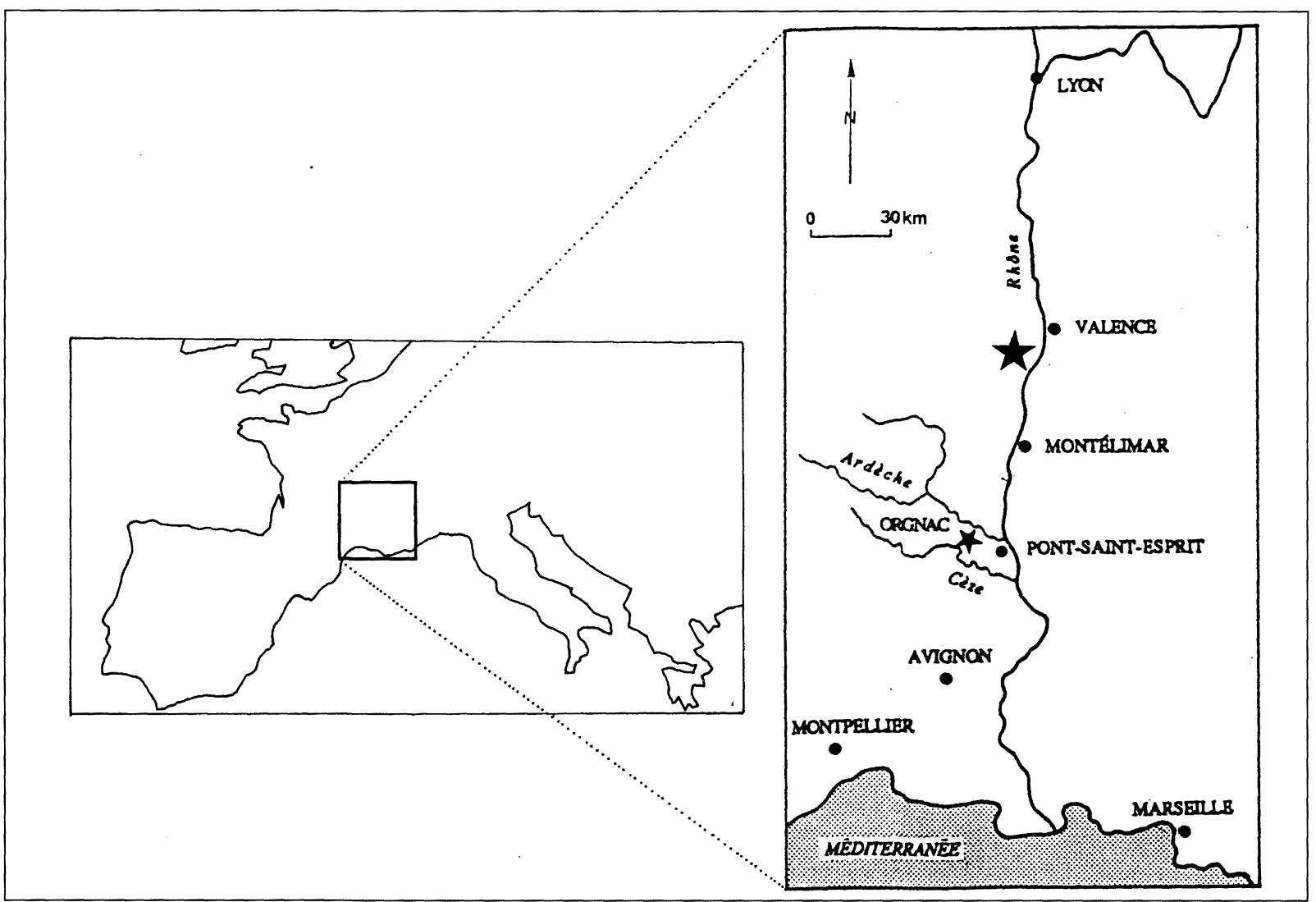

Fig. 1. Localisation du site de Payre (Rompon, Ardèche) dans le sud-est de la France (Moncel).

grottes de Soyons, plus au nord, les autres sites connus à l'heure actuelle, et appartenant au même complexe, sont concentrés le long des gorges de l'Ardèche, plus au sud, et sont datés des stades isotopiques 4 et 3 . Ce gisement a livré plusieurs niveaux d'occupation bien distincts dans le temps, riches en matériel lithique, faunique et en restes humains. Ils permettent de définir d'une part les caractéristiques de groupes humains pré-néandertaliens et néandertaliens ayant circulé dans la vallée du Rhône entre 200000 et 80000 ans, d'autre part les activités pratiquées et la façon dont l'environnement a été exploité. La reconstitution du paléoenvironnement végétal est donc particulièrement interessante pour replacer les allées et venues des hommes dans leur contexte, contexte ayant certainement été un des facteurs favorables à leur installation dans cette zone et cette cavité.

Payre est un complexe karstique étagé connu depuis les années 40 . Plusieurs fouilles ont concerné différentes petites cavités et abris, la plupart chalcolithiques et néolithiques. La zone principale est formée d'un replat rocheux d'environ $80 \mathrm{~m}^{2}$, couvert de sédiments, restes d'une vaste cavité, aujourd'hui effondrée. C'est à partir de 1950 que des sondages sont effectués dans la partie ouest de cette terrasse par J. Combier, E. Beaux, M. SierraSalvado et $\mathrm{L}$. Taupenas qui mettent en évidence une couche archéologique avec matériel lithique et restes osseux abondants dont le Rhinocéros de Merck. Le site devient alors le témoin d'une occupation ancienne pour la région. A partir de 1990, des fouilles programmées par M-H Moncel débutent sur l'ensemble de la terrasse. Elles révèlent plus de $5 \mathrm{~m}$ de remplissage et plusieurs niveaux d'occupation superposés (Moncel, 1993; Moncel et alii, 1993; Moncel, 1996).

\section{LE SITE DE PAYRE}

Le gisement est situé à environ $25 \mathrm{~km}$ au sud de Valence, sur la commune de Rompon, en bordure de la rive droite de la vallée du Rhône, en position de promontoire (Fig. 1). Ce type de situation paraît avoir attiré fortement les Pré-Néandertaliens puis- 


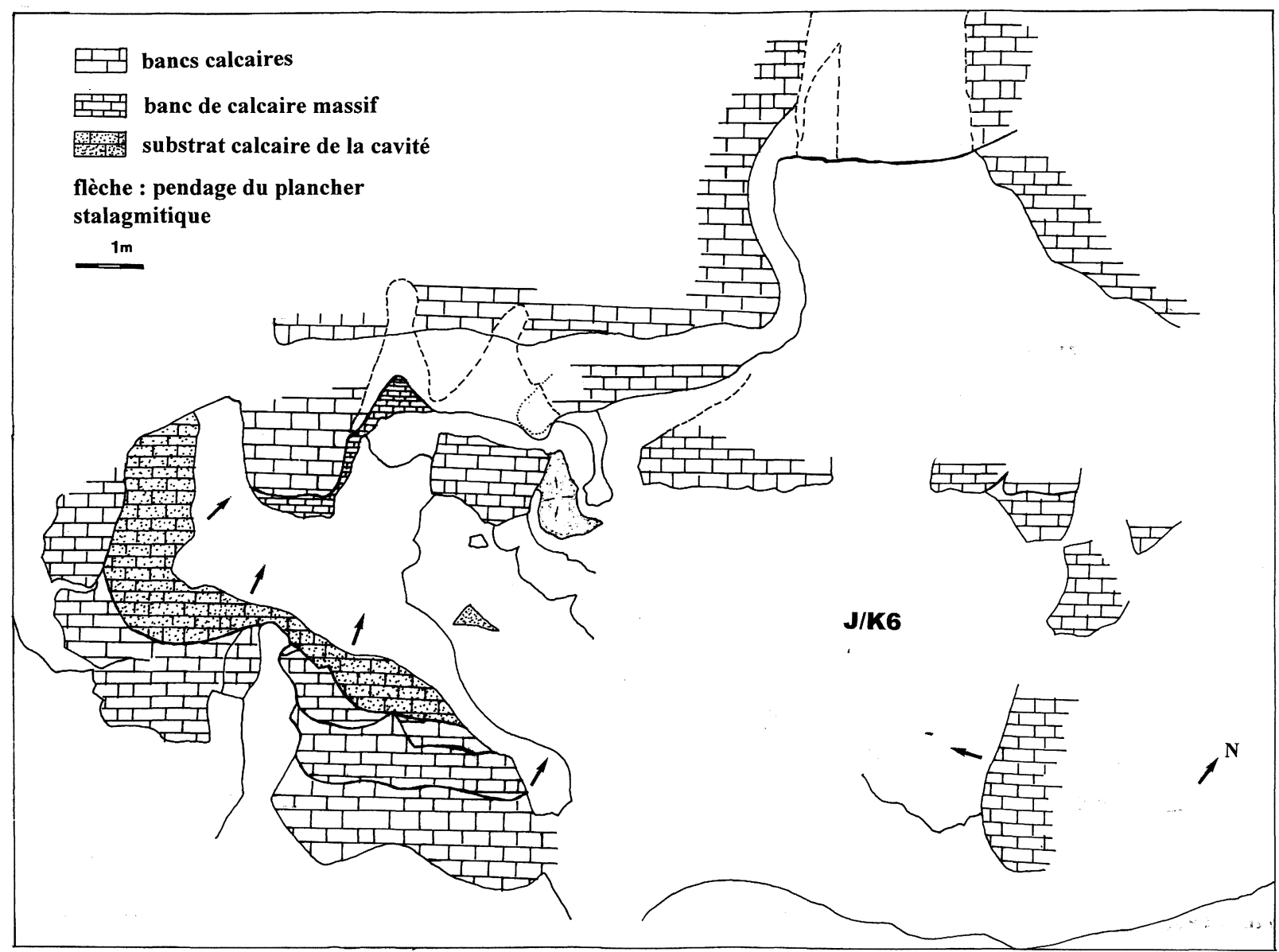

Fig. 2. Plan de la cavité et position de la zone d'échantillonnage pour l'analyse palynologique (Payre, Ardèche) (Moncel, 1999).

qu'elle se retrouve à l'identique pour les grottes de Soyons. Orientée au sud-est, la terrasse est à $60 \mathrm{~m}$ au dessus d'une petite rivière, la Payre, à quelques kilomètres de la confluence avec le Rhône. Cette rivière fait partie d'un réseau fluviatile ouest-est, des contreforts du massif Central à la vallée du Rhône, qui charrie des roches très diverses. Le contexte géologique calcaire (Kimméridgien et Titonique-Portlandien), appartient à un vaste complexe jurassique et crétacé couvrant une grande partie de l'Ardèche méridionale et orientale, à la limite de l'Ardèche cristalline. Le site est localisé sur le rebord d'un plateau calcaire s'élevant à 200-300 m d'altitude, qui forme une des limites occidentales de la vallée du Rhône. La position topographique du lieu est donc particulièrement favorable, à la jonction de plusieurs biotopes (vallées, versants, plateau) et en position d'observatoire. Les hommes sont revenus à plusieurs reprises alors que l'aspect de la cavité s'est modifié au cours du temps.
Les fouilles ont permis de dégager les limites et le volume de la cavité (Fig. 2). Celle-ci était composée d'une salle de $3 \mathrm{~m}$ de hauteur de plafond adjacente à un petit diverticule plus bas $(1,5 \mathrm{~m})$. L'entrée était probablement étroite comme le montrent les ouvertures des autres cavités du secteur. Ce type d'abri a favorisé la fréquentation des ours (Ursus spelaeus) dont les nombreux restes témoignent que la grotte a servi de tanière à ces animaux, morts en hibernation.

\section{CONTEXTE STRATIGRAPHIQUE}

La séquence de $5 \mathrm{~m}$ d'épaisseur, datée par U-Th et ESR sur ossements, dents et plancher stalagmitique (Moncel, 1993; Masaoudi et alii, 1996), peut se résumer comme suit (Fig. 3) :

- dépôt d'un plancher stalagmitique sur les deux bords de la cavité, daté du stade isotopique 7 . 


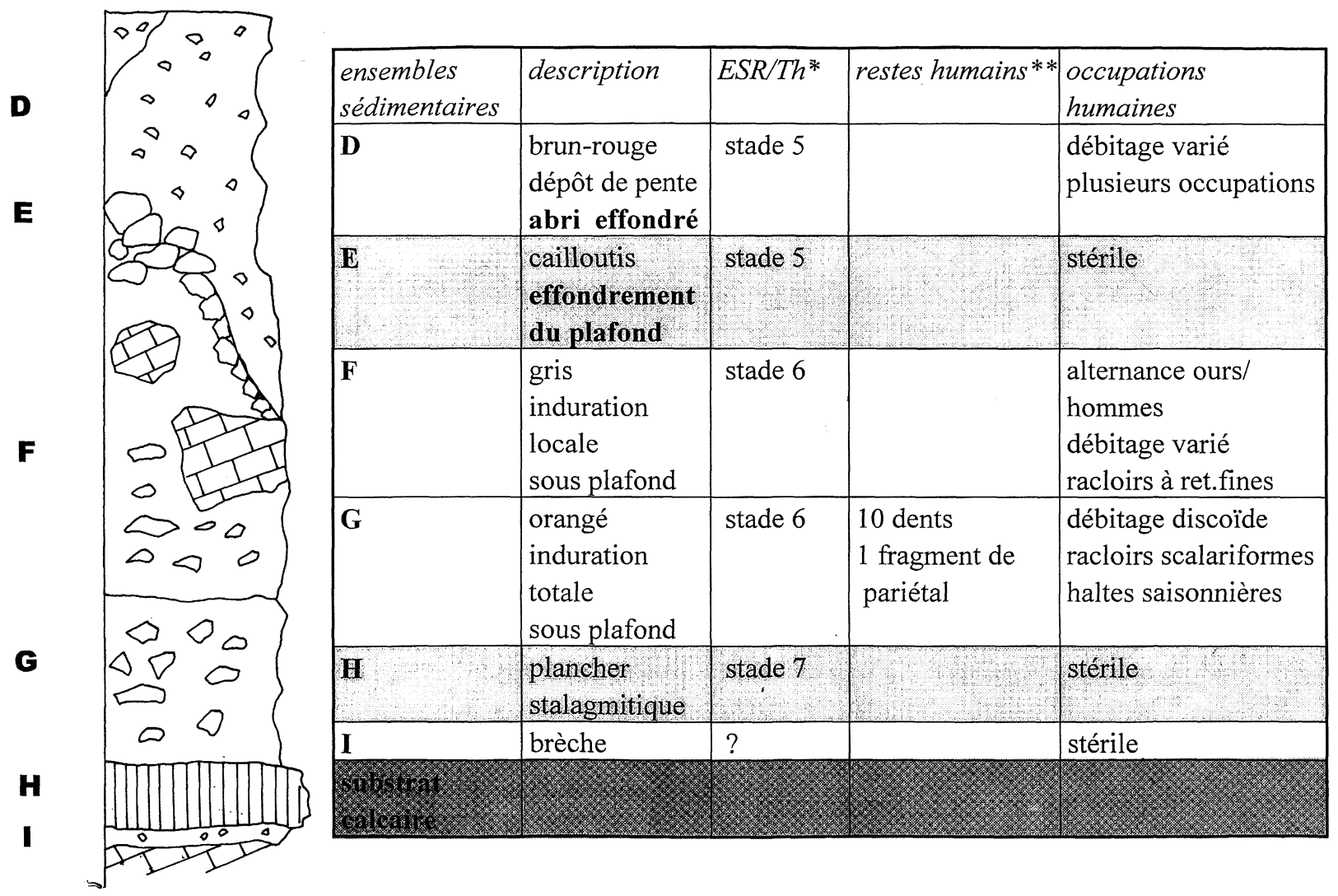

Fig. 3. Tableau synthétique des ensembles sédimentaires, datations et occupations humaines du site de Payre (* Masaoudi et alii, 1996; ** Moncel et Condemi, 1997; Moncel et alii, 1999) (Moncel).

- un premier ensemble (G) argileux orangé et fortement caillouteux, avec des plaquettes et des blocs, se dépose sous abri corrélativement à deux occupations majeures. C'est dans cet ensemble que les rèstes humains sont surtout localisés. Ce sont une dizaine de dents et un fragment de pariétal gauche qui appartiennent à 3 ou 4 individus différents, enfants, adolescents et adultes, tous répartis dans un périmètre restreint et à une même profondeur (Moncel et Condémi, 1996, 1997). Ce dépôt daté du stade isotopique 6 est observable sur la totalité de la surface de la grotte mais a subi une érosion superficielle assez intense. Une forte induration a également affecté les sédiments.

- un second dépôt de même type et de même âge, mais grisâtre $(\mathrm{F})$, se met de nouveau en place dans la grotte corrélativement à deux occupations humaines, en alternance avec de nombreuses fréquentations animales. Une forte induration des sédiments est visible à la jonction de la salle et du diverticule. Elle est due à une arrivée d'eau à partir du plafond.

T. P., 58, n. ${ }^{0} 1,2001$
- un premier éboulement massif du plafond met en contact la grotte avec une autre cavité supérieure au cours du stade isotopique 5 . Son remplissage très caillouteux s'effondre et moule une grande partie des dépôts sous-jacents. Cet ensemble (E) est un amas de cailloutis et de blocs, presque sans emballage sédimentaire et soudé par de la calcite.

- le plafond recule de plus en plus et la dernière grande phase de dépôt s'effectue en partie à l'air libre (ensembles C et D). L'homme fréquente encore le lieu durant le stade isotopique 5. Les ensembles B et A, superficiels, sont des apports sédimentaires du karst aujourd'hui toujours actif et sont stériles.

Il ne subsiste à l'heure actuelle que des lambeaux du toit, au niveau des bancs calcaires sur la falaise.

\section{CONTEXTE ARCHEOLOGIQUE}

Trois grandes phases dans l'occupation humaine ont été clairement identifiées à la fouille. Elle 
sont situées dans les ensembles $\mathrm{G}, \mathrm{F}$ et $\mathrm{D}$, de la base au sommet. Les deux premières se sont déroulées en grotte et la troisième en partie à l'air libre. Quatre lits principaux d'objets sont nettement visibles pour les ensembles $\mathrm{G}$ et $\mathrm{F}$.

La grande homogénéité des comportements de prédation pour tous les niveaux contraste avec la diversité des comportements face à la matière première. Ils sont orientés vers la chasse de Equus caballus, Cervus elaphus et de grands Bovinés et le charognage du Rhinocéros et de jeunes Eléphants (Patou-Mathis et Lamarque in Moncel et alii, 1993, 1999). L'étude taphonomique indique un traitement différentiel des espèces (apport des carcasses entières pour les petits herbivores et de certaines parties seulement pour les plus grands) et des haltes saisonnières en automne, hiver ou printemps. En revanche, le matériel lithique montre des comportements techniques qui diffèrent selon les phases d'occupation. Les hommes ont cependant toujours utilisé en priorité du silex venant d'une zone située à $15 \mathrm{~km}$ au sud et ont récolté dans la rivière en contrebas des galets de basalte et de calcaire pour le façonnage d'outils sur galet. Du quartz et du quartzite ont par ailleurs été débités. Les hommes ont produit des éclats épais (méthode discoïde) dans l'ensemble $\mathrm{G}$ le plus ancien et des éclats plus fins et plus petits dans les deux autres ensembles (débitage Levallois, discoïde, sur éclat). Presque tous les outils sont cependant des racloirs et des pointes. La retouche est envahissante pour l'ensemble $G$ et elle est fine pour les ensembles $F$ et $D$. Deux outils bifaciaux ont par ailleurs été découverts dans le niveau le plus profond, celui-là même où sont les restes humains. Les hommes ont employé le feu (ossements et silex brûlés, lentille cendreuse). Aucune structuration de l'espace domestique n'est toutefois à l'heure actuelle discernable dans les niveaux d'occupation, qui s'étendent sur l'ensemble de l'espace disponible.

L'apport dans le site de blocs entiers de silex venant de gîtes éloignés permet de mesurer indirectement la mobilité des occupants. Les hommes ontils anticipé et préparé leur venue? Le silex local n'est pas d'assez bonne qualité et en plus petits rognons. Le débitage des éclats a eu lieu sur place, pour des activités locales, et le matériel a été ensuite abandonné. Les espèces animales, dont les os portent des marques anthropiques ou sont intensément fracturés, sont tous des herbivores pouvant être chassés ou charognés aux abords du site. Une grande partie de l'outillage paraît du reste avoir été fa- briquée dans cette optique: grands outils sur galets dont le tranchant a été écrasé, percuteurs de grande taille, éclats à longs tranchants en silex et quartzite. L'exploitation ponctuelle d'un environnement favorable et riche paraît l'hypothèse la plus plausible. La diversité des comportements techniques d'une période d'occupation à l'autre ne peut s'expliquer, en l'état actuel des connaissances, par les activités qui ont eu lieu dans la grotte.

Ce Paléolithique moyen ancien est bien représentatif de ce que l'on rencontre dans la région. Il illustre la variété des comportements humains, reflet à la fois des activités et sans doute aussi de groupes de traditions distinctes (Moncel, 1998).

\section{LA VEGETATION ACTUELLE}

La situation géographique et l'environnement géologique de l'Ardèche font que cette région est à la fois sous influence méditerranéenne, atlantique et montagnarde. Les moyennes annuelles de température et des précipitations sont respectivement de l'ordre de $12^{\circ} \mathrm{c}$ et $1000 \mathrm{~mm}$.

Les fonds thermiques établis par C.P. Peguy (1) permettent de situer Payre dans l'étage méditerranéen surplombant l'étage subméditerranéen. Ce gisement se trouve aux adrets sur des terrains calcaires d'où la série d'Yeuse qui constitue la végétation actuelle avec des essences typiques tel que Buis, Thym, Prunier et Lierre. Le long du cours d'eau «la Payre», à $60 \mathrm{~m}$ en contrebas du site, poussent les Platanes, Frênes, Peupliers, Saules, arbres de Judée, Figuiers sauvages, Ronces, Arums, Trèfles, Troènes, Cressons et Chardons. Il existe aussi des plantations d'arbres fruitiers comme l'arbre à Kiwi, le Pommier, le Poirier et le Pêcher.

\section{ÉTUDE POLLINIQUE}

L'étude pollinique a été effectuée le long de plusieurs coupes, dont une principale qui est la J/K6. Les 50 prélèvements concernent la totalité des ensembles stratigraphiques, à savoir, de la base au sommet, G, F, E, D, C, B et A (Fig. 4).

(1) C.P. Peguy, Carte climatique détaillée de la France, Valence. Publication du Bureau de Recherche Géologique et Minière, Centre National de la Recherche Scientifique, France, Equipe de recherche $n^{\circ} 30$ associée à l'Université scientifique et médicale de Grenoble (1972). 


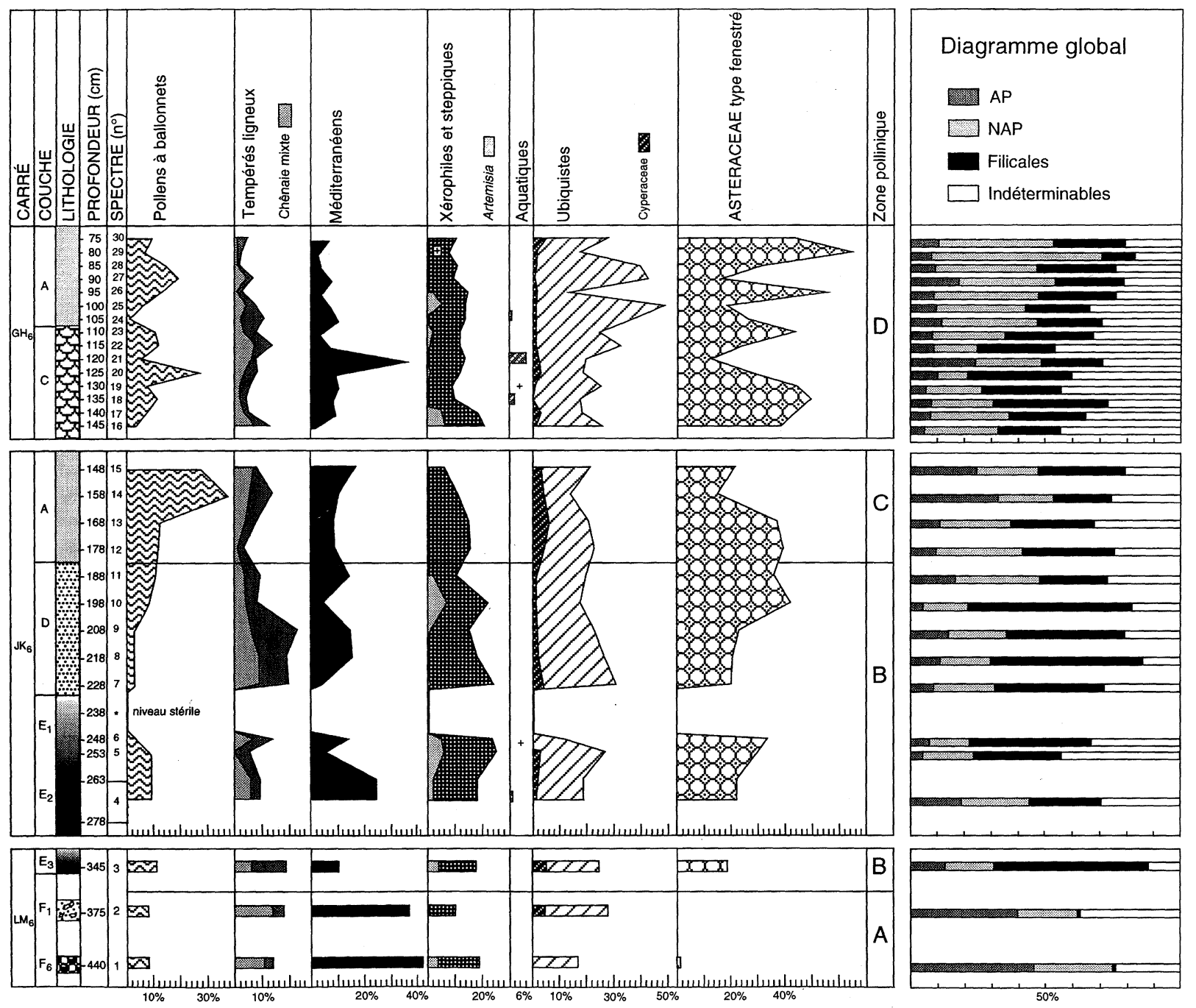

Fig. 4. Diagramme pollinique synthétique du site de Payre (Rompon, Ardèche). Altitude 186 m (Kalaï, 1998).

\section{a/ Méthode d'analyse}

Le sédiment archéologique est soumis à des traitements de base, mécaniques et chimiques, qui consistent à éliminer les fractions minérale et organique non sporo-polliniques. Il est d'abord séché dans l'étuve à $40^{\circ} \mathrm{C}$, ensuite tamisé sur des mailles de 0,5 mm. La quantité de sédiment utilisée est de 20 grammes en moyenne et peut atteindre $40 \mathrm{~g}$ quand le sédiment est très calcaire.

Pour traiter des sédiments minéraux, très peu fossilifères, nous appliquons la méthode chimique classique de C.Sittler (1955) à laquelle est associée une lévigation en liqueur dense: le chlorure de zinc $(\mathrm{d}=2)$.

Au culot final séché, sont ajoutées quelques gouttes de glycérine bidistillée dont l'indice de réfraction est différent de celui de la membrane sporopollinique. On obtient ainsi un montage liquide qui permet de faire tourner les grains pour les observer sous toutes les vues (polaires ou équatoriales).

\section{b/ Présentation des résultats}

Des 50 prélèvements, seuls 30 ont fourni des spectres polliniques valables ( 20 taxons et 100 grains de pollen par niveau). Les résultats obtenus sont représentés ici par un diagramme synthétique (Fig. 4). C'est une représentation globale des variations des associations végétales qui permet de déduire des évolutions paléoécologiques, paléoclima-

T. P., 58, n. ${ }^{\circ} 1,2001$ 
tiques et chronologiques. Ces associations sont constituées des groupes de taxons d'écologie comparable (Suc, 1984; Ablin, 1985; Gauthier, 1992). Nous avons choisi sept groupes.

Groupe 1: les ligneux à pollens à ballonnets sont mis dans un groupe à part étant donné leur grande production et leur excellente dispersion pollinique. Leurs pollens sont présents dans l'atmosphère pendant une grande partie de l'année à cause de leur degré de flottabilité (Cour, 1974) et sont donc susceptibles d'être sur-représentés. Ce groupe comprend Pinus, Pinaceae (tous les pollens à ballonnets qui ne sont pas déterminés au genre), Cedrus, Abies et Picea, les deux derniers taxons exigeant une grande humidité.

Groupe 2: les ligneux tempérés représentés par les taxons «humides et thermophiles» tels que : Acer, Ilex, Hedera, Alnus, Betula, Betulaceae (désignant les grains de pollen de cette famille qui ne sont pas déterminés au genre), Carpinust. betulus, Corylus, Quercus t.pedunculata-pubescens, Castanea, Juglans, Fraxinus, Platanus, Populus, Salix, Taxus, Tilia et Ulmus.

Au sein de ce groupe, se trouve la chênaie mixte avec Quercus t. pedunculata-pubescens, Tilia, Ulmus et Fraxinus mais aussi Carpinus type betulus.

Groupe 3: les méditerranéens ligneux (s. 1.) qui sont Pistacia, Buxus, Quercus t. ilex-coccifera, Cistus, Fraxinus t. ornus, Ligustrum, Olea, Phillyrea et Vitis.

Groupe 4: les AP et NAP xérophiles et steppiques qui comprennent Artemisia, Asteraceae t. échinulé, Centaurea, Caryophyllaceae, Chenopodiaceae, Cupressaceae (Juniperus et Cupressus), Ephedra, Poaceae et Thalictrum.

Groupe 5: les NAP ubiquistes. Il inclut les Apiaceae, Brassicaceae, Campanulaceae, Crassulaceae, Cyperaceae, Ericaceae, Euphorbiaceae, t. Mercurialis, Fabaceae, Lamiaceae, Liliaceae, Polygonum, Rumex, Plantago t. operculé, Plantago, Primulaceae, Ranunculaceae, Resedaceae, Rosaceae, Rubiaceae, Scrofulariaceae, Solanaceae, Urticaceae, Valerianaceae.

Groupe 6: NAP aquatiques ou ensemble des plantes aquatiques comme Potamogeton, ou de milieux humides comme Sparganium.

Groupe 7: lesAsteraceae type fenestré sont isolées en raison de leur importante représentation qui risque de masquer la présence des autres groupes.

Pour le diagramme synthétique, le pourcentage des différents taxons est calculé à partir de la som- me de base qui regroupe les AP (pollens d'arbres, arbustes et arbrisseaux), les NAP (pollens d'herbacées) et les grains indéterminés.

Le diagramme global représenté à l'extrémité droite du schéma synthétique montre l'évolution des différents taux d'arbres, herbacées, Filicales et indéterminables (abimés ou cassés) au travers de la stratigraphie. Pour ce diagramme, le calcul est fait à partir de la somme totale qui regroupe la somme de base, les filicales et les indéterminables.

\section{INTERPRETATION}

Le diagramme pollinique peut être scindé en quatre zones polliniques de la base au sommet (Fig. 4).

Dans la zone pollinique $\mathbf{A}$, les taxons méditerranéens atteignent $42 \%$ à la base grâce au taux de Quercus t. ilex. Les taxons xériques et steppiques sont de l'ordre de $16 \%$ et les taxons ubiquistes compris entre 17 et $28 \%$. Cette zone se caractérise par l'absence des Asteraceae t. fenestré. Le taux de pollen d'herbacées atteint $37 \%$ et celui des arbres $63 \%$.

Cette zone correspond à l'ensemble stratigraphique F. Elle témoigne d'un paysage de type semiforestier avec une végétation méditerranéenne dominée par Quercus t. ilex.

Dans la zone pollinique $B$, le taux d'arbres a diminué (20 à 44\%). Cette chute des arbres est due surtout à celle de Quercus t. ilex qui passe de 37 à $7 \%$. Par conséquent, les taxons méditerranéens sont moins importants $(17 \%)$. On note néanmoins la présence de taxons thermophiles comme Hedera, Juglans, Carpinus t. betulus, Castanea, Buxus et Populus.

Les Asteraceae t. fenestré sont abondantes (17 à $42 \%$ ) de même que les xérophiles (11 à 24\%), notamment les Poaceae, les Cupressaceae, Artemisia et Centaurea. A partir de cette zone, de rares indices de plantes de milieu marécageux ou aquatique sont observés.

Cette zone correspond aux ensembles stratigraphiques $\mathrm{E}$ et $\mathrm{D}$ qui se sont donc déposés au sein d'un milieu semi-ouvert où les Asteraceae et les Poaceae dominent les herbacées. La base de cette zone est caractérisée par Quercus t. ilex dont les taux reculent au sommet au profit de Buxus. La présence de taxons tempérés comme Hedera, Quercus t. pedunculata, Tilia et Alnus suggère un climat tempéré. Cependant, la présence de Quercus t. ilex, 
Buxus, Pistaciaet Cistus souligne l'influence méditerranéenne.

La zone pollinique $\mathbf{C}$ se distingue par l'importance du taux de pollens à ballonnets (28 à $38 \%$ ), par la baisse desAsteraceae t. fenestré, des xériques et par l'absence de Artemisia. En revanche, les Cyperaceae et les Brassicaceae enregistrent des valeurs de l'ordre de $6 \%$.

La végétation semble être de type semi-ouvert et marquée par le développement de Pinus et la présence de Picea. Ce léger changement suggère un raffraîchissement climatique.

Dans lazone pollinique $\mathbf{D}$ les taxons ubiquistes atteignent $48 \%$ par l'abondance des Brassicaceae. Les Asteraceae t. fenestré (13-64\%) et les taxons xérophiles (13-22\%) sont prédominants par rapport aux taxons méditerranéens et tempérés (7\%). Le groupe des taxons aquatiques atteint $6 \%$ à la base de cette zone.

La présence des Cupressaceae, Lamiaceae, Brassicaceae et Asteraceae laisse suggérer un milieu ouvert et un climat plus sec. La richesse en Brassicaceae est certainement locale car nous n'avons trouvé aucun autre enregistrement pollinique comparable.

\section{CONCLUSION}

L'enregistrement de la pluie pollinique fossile à Payre est caractérisé dans son ensemble par une grande homogénéité taxonomique qui ne permet pas de définir une véritable dynamique de végétation. Deux hypothèses ont été émises pour tenter d'expliquer ce phénomène : soit une rapidité de la vitesse du dépôt (qui est plausible dans un contexte karstique actif), soit des conditions climatiques stables durant une longue période. L'abondance des Filicales indique un apport sédimentaire par ruissellement pouvant être une cause de la rapidité et de la puissance du dépôt, contrairement aux conditions de dépôt de l'ensemble F.

Les échantillons de l'ensemble $\mathrm{G}$ (à la base) et ceux de l'ensemble B (au sommet) n'ont apporté aucune information en raison de la stérilité du sédiment en matériel sporo-pollinique.

Le paysage est donc généralement semi-ouvert avec des taxons arboréens omniprésents comme Quercus t. ilex et Buxus. Le climat est de type tempéré sous influence méditerranéenne (Kalaï, 1998).

Alors que les grands mammifères signalent une certaine stabilité des faunes (interférence des choix humains?), les micromammifères en revanche montrent des variations stratigraphiques plus marquées, en particulier entre les ensembles $\mathrm{G}$ et $\mathrm{F}$, et l'ensemble D. La présence de Microtus arvalis dans toutes les couches et la disparition de Microtus gregalis au sommet de l'ensemble F indiqueraient un climat aride et relativement froid pour l'ensemble G. Le climat deviendrait moins rigoureux vers le sommet de l'ensemble $\mathrm{F}$ pour être franchement méditerranéen et humide en D (El Hazzazi, 1998; Moncel et alii, 1999).

L'enregistrement pollinique de l'ensemble D est en accord avec la plupart des données des autres études, et donc un bon indicateur, parmi d'autres, pour évoquer le cadre environnemental de la cavité lors du stade isotopique 5. La différence des données polliniques avec celles des micro-mammifères pour l'ensemble $F$ pourrait être due aux conditions d'enfouissement et de mise en place. L'entrée étroite de la cavité, à certains moments, a probablement pu isoler en partie l'intérieur de la grotte du monde extérieur.

Quoi qu'il en soit, l'analyse pollinique des trois quart de cette séquence est une étape dans la connaissance des données climatiques de cette région méridionale de la France. Que les conditions climatiques aient amplement ou modéremment varié au cours du temps, les hommes ont exploité l'environnement faunique de la même manière. La position du site est alors à considérer comme un facteur déterminant dans le choix de certains comportements de subsistance.

\section{BIBLIOGRAPHIE}

Ablin, D. (1985): Analyse pollinique des dépôts lacustres de Ceyssac (Plio-Pléistocène du Velay, Massif central français): flore, végétation, climat. Thèse, Université Pierre et MarieCurie, Paris VI et M.N.H.N. Paris.

Cour, P. (1974): "Nouvelles techniques de détection des flux et des retombées polliniques: étude de la sédimentation des pollens et des spores à la surface du sol". Pollen et Spores, 16, 1: 103-141.

El HAZZAZI, N. (1998): Paléoenvironnement et chronologie des sites du Pléistocène moyen et supérieur Orgnac 3, Payre et Abri des Pêcheurs (Ardèche, France) d'après l'étude des rongeurs. Thèse de doctorat du Museum National d'Histoire Naturelle. Paris.

Gauthier, A. (1992): Paléoenvironnements du Pléistocène moyen dans le sud de la France. Apport et limites de l'analyse pollinique de trois sites préhistoriques: Caune de l'Arago, Orgnac 3, Grotte du Lazaret. Thè-

T. P., 58, n. ${ }^{\circ} 1,2001$ 
se de doctorat du Muséum National d'Histoire Naturelle. Paris.

KaLAÏ, C. (1998): Reconstitution du paléoenvironnement végétal et du paléoclimat de la fin du Pléistocène moyen et du Pléistocène supérieurd'après les analyses polliniques de la Baume Moula-Guercy, du site de Payre et de l'abrides Pêcheurs (Ardèche, France). Thèse de doctorat du Muséum Nationald'Histoire Naturelle. Paris.

Masaoudi, H.; Falgueres, C.; Bahain, J.-J. et Moncel, M.H. (1996): "Datation du site Paléolithique moyen de Payre (Ardèche): nouvelles données radiométriques (méthodes U/Th et ESR)". Compte Rendu de l'Académie des Sciences. Paris, 324, série IIa: 149-156.

Moncel, M.-H. (1993): "Le site de Payre (commune de Rompon, Ardèche): une occupation humaine du Paléolithique moyen ancien". Quaternaire, 4: 149-157.

- (1996): "Une nouvelle industrie lithique du Paléolithique moyen ancien: le site de Payre (Ardèche, France)". Compte Rendu de l'Académie des Sciences, 323, série IIa: 275-282.

- (1998): "Le Paléolithique moyen de la moyenne vallée du Rhône en France: la question de la variabilité des assemblages lithiques des stades isotopiques 9 à 3". Anthropologie, XXXVI/3: 181-199. Brno.

Moncel, M.-H.; Bahain, J.-J.; Falgueres, C.; Hazzazi, El N.; KalaÏ, C.; Masaoudi, H.; Muahad, M.; Patou-Mathis, M. et Renault-Miskovsky, J. (1993): "Le site de Payre (commune de Rompon, Ardèche): un site paléo- lithique moyen ancien dans un contexte d'abri effondré. Premier bilan des études pluridisciplinaires, position chronologique, paléoenvironnement, paléoclimatologie". Quaternaire, 4: 159-173.

Moncel, M.-H. et Condemi, S. (1996): “Découverte de dents humaines dans le site paléolithique moyen de Payre (Ardèche, France)". Compte Rendu de l'Académie des Sciences, 322, série IIa: 251-257.

- (1997): "Des restes humains dans le site paléolithique moyen ancien de Payre (Ardèche): dents et pariétal". Bulletin de la Société Préhistorique Française, 94, 2: 168-172.

Moncel, M.-H.; Bahain, J.-J.; Bocherens, H.; Condemi, S.; Desclaux, E.; Drucker, D.; Dubar, M.; Falgueres, C.; Froget, L.; Hazzazi, El N.; Kalaï, C.; Lamarque, F.; Masaoudi, H.; Mercier, N.; Patou-Mathis, M.; TheryParisot, I. et Valladas, H. (1999): "Le site de Payre (Rompon, Ardèche). Paléolithique moyen et Néandertaliens. Bilan des fouilles programmées 1990-1999". Service Régional de l'Archéologie Rhône-Alpes. Ministère de la Culture. Lyon.

SitTLER, C. (1955): "Méthodes et techniques physico-chimiques de préparation des sédiments en vue de leur analyse pollinique". Revue Institut Français du Pétrole, $X, 2: 103-114$.

Suc, J.P. (1984): "Origin and evolution of the mediterranean vegetation and climate in Europe". Nature, 307, 5950: 429-432. 